\title{
Zeiform mouthbrooding in the deepsea - dispelling a myth
}

\author{
Ralf Britz $^{1 *}$, G. David Johnson ${ }^{2} \&$ Kevin W. Conway ${ }^{3}$
}

${ }^{1}$ Senckenberg Natural History Collections Dresden, Museum of Zoology, Dresden 01109, Germany. ${ }^{2}$ Smithsonian Institution, National Museum of Natural History, Vertebrate Zoology, Division of Fishes, Washington D.C. 20560, USA. ${ }^{3}$ Department of Ecology and Conservation Biology and Biodiversity Research and Teaching Collections, Texas A\&M University, College Station, Texas 77843-2258, USA. *email: ralf.britz@ senckenberg.de

Mouthbrooding or oral incubation, the retention of early developmental stages inside of the mouth for an extended period of time, has evolved multiple times in bony fishes ${ }^{1,2}$. Though uncommon, this form of parental care has been documented and well-studied in several groups of freshwater fishes but is also known to occur in a small number of marine fishes, all inhabiting coastal waters ${ }^{1,2}$. A recent paper $^{3}$, reported for the first time mouthbrooding in a deep-water fish species, the zeiform Parazen pacificus, which according to the authors "fills in a gap in the larval literature for this family of fishes and prompts further investigation into other novel reproductive modes of deep-sea fauna."

Singer et al.'s $2020^{3}$ conclusion of mouth brooding in Parazen was based on a single specimen of $104.3 \mathrm{~mm}$ length, which was obtained from a market in Taiwan. As documented in their paper $^{3}$, this specimen has a large egg mass lodged between and extending forward from its pharyngeal jaws and another, smaller egg mass below the gill arches anterior to the basihyal ('tongue' of Singer et al. $2020^{3}$ ). The number of eggs was estimated to be around 530 with an average diameter of $0.73 \mathrm{~mm}$. The authors reported "tendril structures...extending off the portion of the tongue and palate" that "seemed to be present to hold the egg mass in place". The embryos in the eggs "had developed eyes, a notochord and a visible yolk sac, but had no pared (sic) fins." In their discussion, they stated that "There is no way to tell for sure if the eggs in this specimen are Parazen pacificus given that there has not been a formal description of its eggs or larva." It is exactly this sentence that led us to question the conclusion of Singer et al. $2020^{3}$ and to look further into the identity of the eggs that the authors found in the mouth of their specimen. Given the potential implications of their discovery, we wonder why the authors chose not to examine the embryos more closely. When zooming in on the eggs illustrated in their figures 4 and 5 we observed several structures inside the eggs that we wanted to explore further. We therefore imported the figures from the published PDF into Photoshop and enhanced the contrast by using the program's ImageAdjustment-Levels function. This made several details of the embryos visible more clearly. We observed a number of segmented limbs in three of the embryos in figure 5, which made us suspect that the eggs are not fish eggs, but most likely eggs of crustaceans. We then searched for images of crustacean eggs and found photos of the eggs of Blue (Paralithodes) ${ }^{4}$ and Red King Crab (Lithodes) $)^{5}$ that resemble the eggs in Singer et al.'s 2020 figure 5 closely in appearance and size. The King Crab embryos have a large yolk sac, larval swimming limbs (maxillipeds) stacked up around the outline of the yolk sac, elliptical compound eyes and a segmented tail ${ }^{4,5}$. In the enhanced photo (Fig. 1) of the eggs in figure 5 of Singer et al. 2020 the elliptical eyes, the swimming limbs and the segmented tail ('notochord' of Singer et al. 2020) can be recognized. The egg masses of crabs and those of other decapods are held together by tendril-like structures ${ }^{6,7}$, another fact in line with our identification of the eggs in Singer et al.'s 2020 specimen of Parazen as crab eggs. We are therefore convinced that the egg mass in Singer et al.'s 2020 figures are decapod crustacean eggs, most likely the eggs of a brachyuran crab and potentially those of a member of the family Lithodidae, which are 
common around the coast of Taiwan ${ }^{7}$. We conclude by stressing that mouth brooding is restricted to a few shallow water marine fish groups and that it is still undocumented in bony fishes inhabiting the deep sea.

1. Oppenheimer, J.R. Mouthbreeding in fishes. Animal Behaviour. 18(3), 493-503 (1970).

2. Blumer, L.S. A bibliography and categorization of bony fishes exhibiting parental care. Zoological Journal of the Linnean Society. 75(1), 1-22 (1982).

3. Singer, R.A., Moore, J.A. \& Stanley, E.L. Novel life history strategy in a deep sea fish challenges assumptions about reproduction in extreme environments. Scientific Reports. 10, 3649 (2020). https://doi.org/10.1038/s41598-020-60534-0

4. Stevens, B.G. Embryo development and morphometry in the blue king crab Paralithodes platypus studied by using image and cluster analysis. Journal of Shellfish Research. 25(2), 569-576 (2006).

5. http://www.sitnews.us/0207news/020707/020707_crab_eggs.html

6. Cheung, T.S. The development of egg-membranes and egg attachment in the shore crab, Carcinus maenas, and some related decapods. Journal of the Marine Biological Association of the United Kingdom. 46(2), 373-400 (1966).

7. Goudeau, M., Talbot, P., \& Harper, R. Mechanism of egg attachment stalk formation in the lobster, Homarus. Gamete Research. 18(4), 279-289 (1987).

8. Ahyong, S.T., \& Chan, T.Y. Lithodes formosae, a new species of king crab from Taiwan (Crustacea: Decapoda: Lithodidae). Zootaxa, 2332(8), 61-68 (2010).

\section{Acknowledgements}

We thank Bradley Stevens, University of Maryland Eastern Shore, for permission to use the photo of the developing Paralithodes, and Rafael Lemaitre, Smithsonian Institution, National Museum of Natural History, Washington D.C. and Peter K. L. Ng, Lee Kong Chian Museum of Natural History, Singapore, for confirming our identification of the eggs in Singer et al. 2020 as crustacean, most likely crab eggs.

\section{Author contributions}

R. Britz and K. W. Conway conceived the idea for the paper and wrote the first draft. All authors commented on the draft and reviewed the manuscript.

\section{Competing interests}

The authors have no competing interests. 


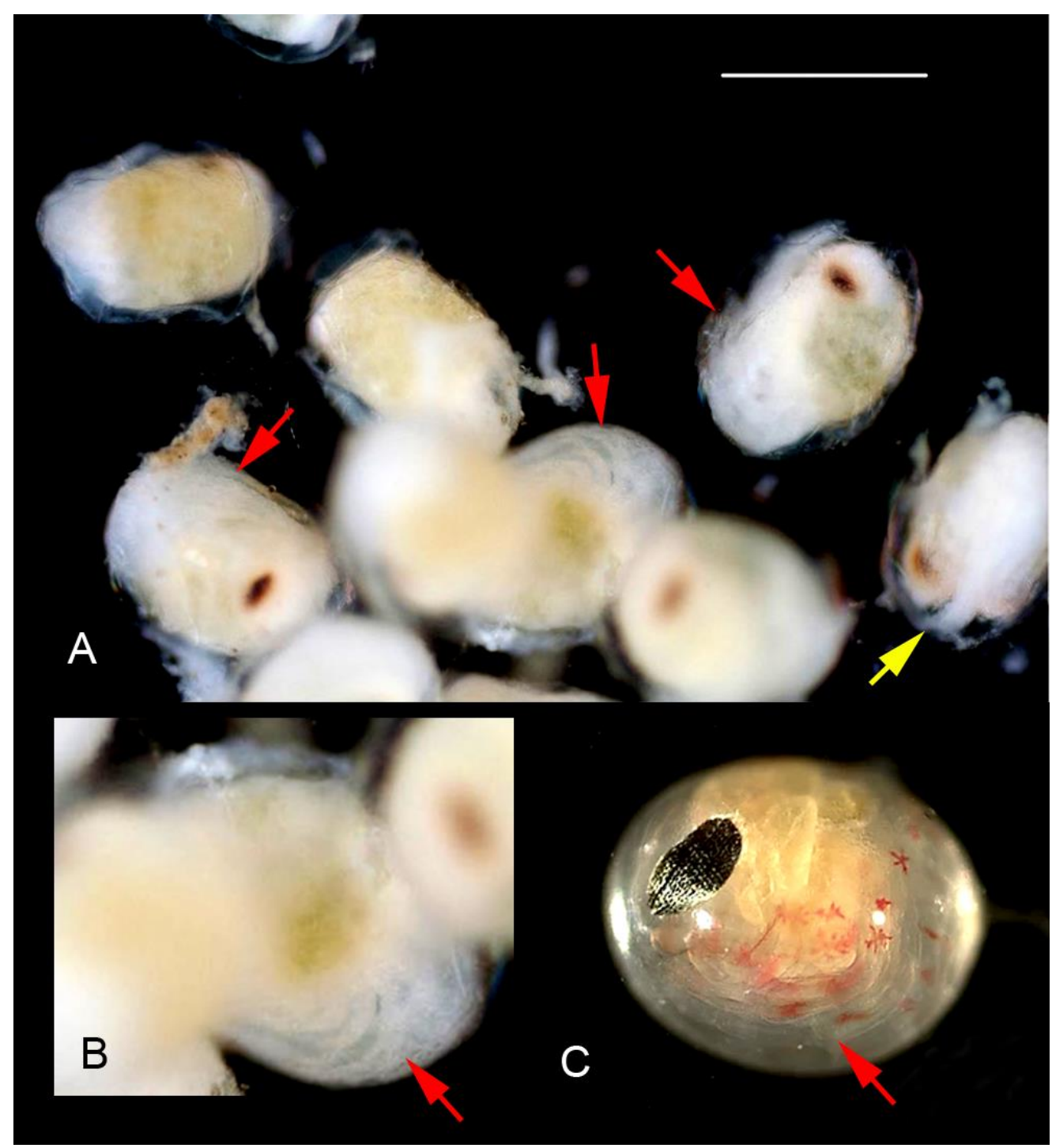

Figure 1. Comparison of embryo containing eggs from the mouth cavity of Parazen pacificus and those of a brachyuran decapod. (A) Reproduction of Singer et al.'s figure 5 with enhanced contrast. (B) Individual egg, image flipped vertically and enlarged. (C) Egg of Blue King Crab (Paralithodes platypus) with developing embryo at day 409 after fertilization (courtesy of Bradley Stevens). Red arrows mark larval limbs in (A-C), yellow arrow marks segmented tail in (A). Note close similarity in elliptical shape of eye and presence of limbs in embryos recovered from the mouth of Parazen pacificus and the Paralithodes embryo. 